Acta Crystallographica Section E

\section{Structure Reports}

Online

ISSN 1600-5368

\title{
3,7-Bis(dimethylamino)phenothiazin-5-ium nitrate dihydrate
}

The reaction of 3,7-bis(dimethylamino)phenothiazin-5-ium chloride pentahydrate (methylene blue) with silver nitrate in a 1:2 molar ratio in water yielded the title compound, $\mathrm{C}_{16} \mathrm{H}_{18} \mathrm{~N}_{3} \mathrm{~S}^{+} \cdot \mathrm{NO}_{3}{ }^{-} \cdot 2 \mathrm{H}_{2} \mathrm{O}$, as one of the products. The cationic dye molecules are planar and stacked in an antiparallel fashion, exhibiting $\pi-\pi$ associations at a distance of 3.7040 (18) $\AA$. The nitrate anion and the two water molecules are involved in a hydrogen-bonding network that also includes the phenothiazinium $\mathrm{N}$ atom.

\section{Comment}

Methylene blue (MB) is an important cationic dye with various colorimetric uses (Tuite \& Kelly, 1993). It is marketed as its chloride salt. Other forms, such as the cyanide and nitrate [the title compound, (I)] salts exhibit antimethemoglobinemic, antiseptic and disinfectant properties (The Merck Index, 2001). The Cambridge Structural Database (Version 5.25, April 2004 update; Allen, 2002) includes four MB crystal structures containing the phenothiazinium moiety, as chloride pentahydrate (Marr et al., 1973), triiodide (Endres et al., 1977), thiocyanate (Kahn-Harari et al., 1973), urate hexahydrate (Sours et al., 2002) and bis(maleonitriledithiolato)cuprate(II) (Snaathorst et al., 1981) salts. In each case, the bond distances in the MB cations indicate a resonance structure in which the positive charge is delocalized over the two dimethylamine $\mathrm{N}$ atoms, with conjugation through the phenothiazinium $\mathrm{N}$ atom, although the $\mathrm{MB}$ cation is sometimes represented as aromatic with a positive $\mathrm{S}$ atom. The addition of $\mathrm{AgNO}_{3}$ to aqueous $\mathrm{MB}$ chloride solution does not precipitate silver chloride, yet the IR spectrum of the product shows features very different from that of the starting materials. Further investigations are currently underway to determine the nature of the resultant soluble silver product but here we report the structure of the precipitated product.<smiles>CN(C)c1ccc2nc3ccc(=[N+](C)C)cc-3sc2c1</smiles>

(I)

The asymmetric unit of (I) consists of an MB cation, a nitrate anion and two water molecules (Fig. 1). The MB cation displays a typical $\mathrm{MB}$ resonance structure, as evident from the pronounced shortening of the $\mathrm{C} 1-\mathrm{C} 2, \mathrm{C} 4-\mathrm{C} 12, \mathrm{C} 8-\mathrm{C} 9$ and $\mathrm{C} 6-\mathrm{C} 13$ bonds compared with other $\mathrm{C}-\mathrm{C}$ bonds (Table 1). The two $\mathrm{C}-\mathrm{S}$ bonds are equal in length, indicating that
Received 24 June 2004 Accepted 14 July 2004 Online 24 July 2004 


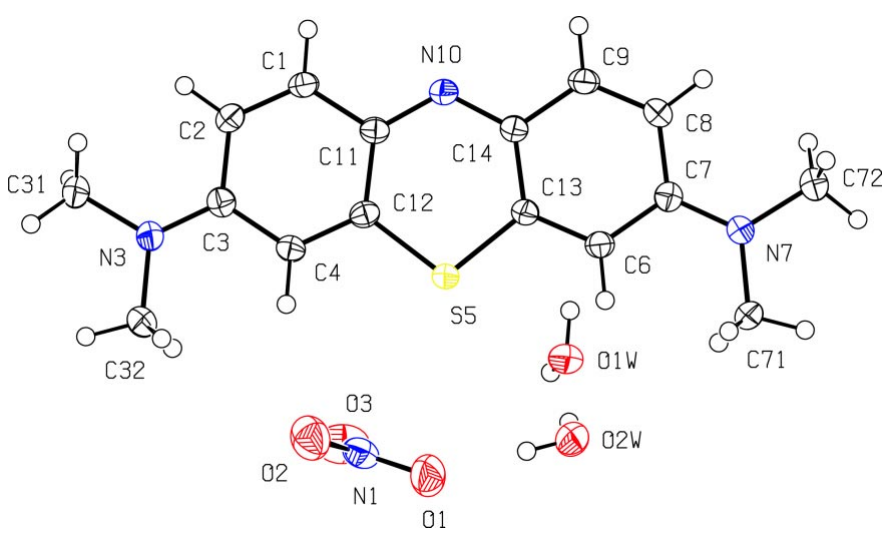

Figure 1

Molecular structure and atom-numbering scheme for (I). Displacement ellipsoids are drawn at the $50 \%$ probability level.

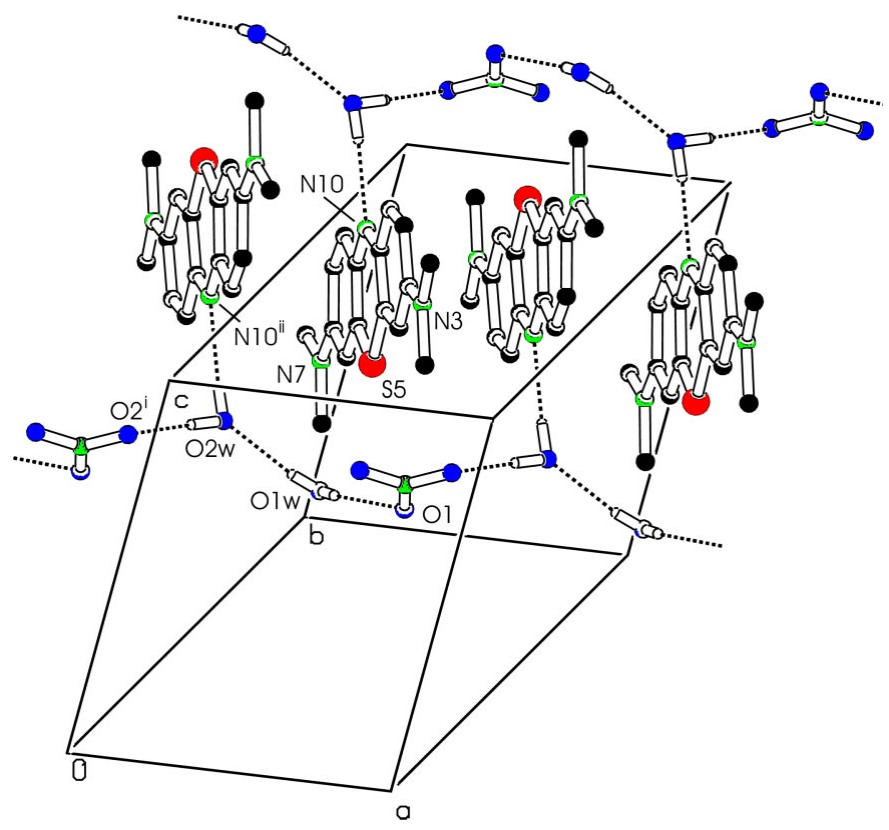

Figure 2

Packing diagram for (I). For clarity, the non-essential $\mathrm{H}$ atoms have been omitted. [Symmetry codes: (i) $-1+x, y, z$; (ii) $-x,-y+1,-z+2$.]

conjugation occurs via $\mathrm{N} 10$. The MB cation is thus planar and the cations are stacked in an antiparallel fashion, exhibiting $\pi-$ $\pi$ associations at a distance of 3.7040 (18) $\AA$. In contrast to MB chloride, which crystallizes with five lattice water molecules, the degree of hydration of the MB nitrate is lower. This difference may be due in part to the hydrogen-bonding network and the fact that more hydrogen-bond acceptor atoms are available on the nitrate ion than for a chloride anion. Hydrogen-bonding associations are listed in Table 2 and indicate that all of the strong hydrogen-bond donor atoms are utilized, although this is not the case for the strong hydrogen-bond acceptor atoms. Only one hydrogen-bond acceptor atom from the phenothiazinium moiety, N10, is involved in the hydrogen-bond network, along with two nitrate $\mathrm{O}$ atoms and one water $\mathrm{O}$ atom. The nitrate ion and the water molecules form a one-dimensional hydrogen-bonded chain in the $a$ cell direction (Fig. 2). There are close contacts between the $\mathrm{S}$ atom and the nitrate $\mathrm{N}$ atom $[3.369$ (4) $\AA$ ] and also between atom O3 and both N3 [2.906 (4) $\AA$; symmetry code: $(1-x,-y, 2-z)]$ and C32 [2.962 (4) $\AA$; symmetry code: $(1-x,-y, 2-z)]$, although for the latter there is no appropriate $\mathrm{H}$ atom to construct a $\mathrm{C}-\mathrm{H} \cdots \mathrm{O}$ association. These close contacts for $\mathrm{O} 3$ may explain why there is no strong hydrogen-bonding interaction with this atom. Two unassigned areas of electron density, 0.64 and $0.59 \mathrm{e} \AA^{-3}$, exist approximately equidistant, 1.94 and $1.96 \AA$, respectively, from atom $\mathrm{S} 5$, which may be the cause of the higher than expected $R$ values.

\section{Experimental}

Methylene blue ( $1 \mathrm{mmol}$ ) was dissolved with silver nitrate $(2 \mathrm{mmol})$ in water $(10 \mathrm{ml})$. Crystals of the title compound were obtained by the slow evaporation of this reaction mixture.

Crystal data

$\mathrm{C}_{16} \mathrm{H}_{18} \mathrm{~N}_{3} \mathrm{~S}^{+} \cdot \mathrm{NO}_{3}{ }^{-} \cdot 2 \mathrm{H}_{2} \mathrm{O}$

$M_{r}=382.44$

Triclinic, $P \overline{1}$

$a=7.6985(3) \AA$

$b=10.9638(3) \AA$

$c=11.3244(4) \AA$

$\alpha=87.081(2)^{\circ}$

$\beta=76.032(2)^{\circ}$

$\gamma=73.524(2)^{\circ}$

$V=889.33(5) \AA^{3}$

$Z=2$

$D_{x}=1.428 \mathrm{Mg} \mathrm{m}^{-3}$

Mo $K \alpha$ radiation

Cell parameters from 12187

reflections

$\theta=2.9-27.5^{\circ}$

$\mu=0.22 \mathrm{~mm}^{-1}$

$T=120(2) \mathrm{K}$

Prism, red

$0.42 \times 0.36 \times 0.20 \mathrm{~mm}$

Data collection

Nonius KappaCCD area-detector diffractometer

$\varphi$ and $\omega$ scans

Absorption correction: multi-scan (SORTAV; Blessing, 1995)

$T_{\min }=0.836, T_{\max }=0.952$

17489 measured reflections

Refinement

Refinement on $F^{2}$

$R\left[F^{2}>2 \sigma\left(F^{2}\right)\right]=0.068$

$w R\left(F^{2}\right)=0.206$

$S=1.13$

4077 reflections

255 parameters

$\mathrm{H}$ atoms treated by a mixture of independent and constrained refinement

4077 independent reflections 3225 reflections with $I>2 \sigma(I)$ $R_{\text {int }}=0.068$

$\theta_{\text {max }}=27.6^{\circ}$

$h=-9 \rightarrow 9$

$k=-14 \rightarrow 14$

$l=-14 \rightarrow 14$

Table 1

Selected bond lengths (§).

\begin{tabular}{llll}
\hline $\mathrm{C} 1-\mathrm{C} 2$ & $1.359(4)$ & $\mathrm{C} 6-\mathrm{C} 7$ & $1.415(4)$ \\
$\mathrm{C} 1-\mathrm{C} 11$ & $1.430(4)$ & $\mathrm{C} 7-\mathrm{N} 7$ & $1.340(4)$ \\
$\mathrm{C} 2-\mathrm{C} 3$ & $1.439(4)$ & $\mathrm{C} 7-\mathrm{C} 8$ & $1.436(4)$ \\
$\mathrm{C} 3-\mathrm{N} 3$ & $1.335(4)$ & $\mathrm{C} 8-\mathrm{C} 9$ & $1.361(4)$ \\
$\mathrm{C} 3-\mathrm{C} 4$ & $1.416(4)$ & $\mathrm{C} 9-\mathrm{C} 14$ & $1.424(4)$ \\
$\mathrm{C} 4-\mathrm{C} 12$ & $1.373(4)$ & $\mathrm{N} 10-\mathrm{C} 11$ & $1.335(4)$ \\
$\mathrm{S} 5-\mathrm{C} 13$ & $1.728(3)$ & $\mathrm{N} 10-\mathrm{C} 14$ & $1.344(4)$ \\
$\mathrm{S} 5-\mathrm{C} 12$ & $1.728(3)$ & $\mathrm{C} 11-\mathrm{C} 12$ & $1.438(4)$ \\
$\mathrm{C} 6-\mathrm{C} 13$ & $1.376(4)$ & $\mathrm{C} 13-\mathrm{C} 14$ & $1.432(4)$ \\
\hline
\end{tabular}


Table 2

Hydrogen-bonding geometry $\left(\AA{ }^{\circ}\right)$.

\begin{tabular}{lllll}
\hline$D-\mathrm{H} \cdots A$ & $D-\mathrm{H}$ & $\mathrm{H} \cdots A$ & $D \cdots A$ & $D-\mathrm{H} \cdots A$ \\
\hline $\mathrm{O} 1 W-\mathrm{H} 11 W \cdots \mathrm{O} 2^{\mathrm{i}}$ & $0.88(5)$ & $1.91(5)$ & $2.789(4)$ & $174(4)$ \\
$\mathrm{O} 1 W-\mathrm{H} 12 W \cdots \mathrm{N} 10^{\mathrm{ii}}$ & $0.84(5)$ & $2.10(5)$ & $2.929(4)$ & $172(4)$ \\
$\mathrm{O} 2 W-\mathrm{H} 21 W \cdots \mathrm{O} 1$ & $0.81(6)$ & $2.14(6)$ & $2.886(5)$ & $154(5)$ \\
$\mathrm{O} 2 W-\mathrm{H} 22 W \cdots \mathrm{O} 1 W$ & $0.75(5)$ & $2.05(5)$ & $2.792(4)$ & $171(5)$ \\
\hline
\end{tabular}

Symmetry codes: (i) $x-1, y, z$; (ii) $-x, 1-y, 2-z$.

All $\mathrm{H}$ atoms, except the water $\mathrm{H}$ atoms, were included in the refinement at calculated positions, in the riding-model approximation, with $\mathrm{C}-\mathrm{H}$ distances of 0.95 (aromatic $\mathrm{H}$ atoms) and $0.98 \AA$ $\left(\mathrm{CH}_{3} \mathrm{H}\right.$ atoms $)$. The isotropic displacement parameters were set equal to $1.25 U_{\text {eq }}$ of the carrier atom. The water $\mathrm{H}$ atoms were located in difference syntheses and both positional and displacement parameters were refined.

Data collection: DENZO (Otwinowski \& Minor, 1997) and COLLECT (Hooft, 1998); cell refinement: DENZO and COLLECT; data reduction: $D E N Z O$, SCALEPACK (Otwinowski \& Minor, 1997) and COLLECT; program(s) used to solve structure: SHELXS97 (Sheldrick, 1997); program(s) used to refine structure: SHELXL97 (Sheldrick, 1997); molecular graphics: PLATON97
(Spek, 1997); software used to prepare material for publication: SHELXL97.

The authors thank the EPSRC National Crystallography Service (Southampton, England).

\section{References}

Allen, F. H. (2002). Acta Cryst. B58, 380-388.

Blessing, R. H. (1995). Acta Cryst. A51, 33-38.

Endres, H., Jeromin, G. \& Keller, H. J. (1977). Z. Naturforsch. Teil B, 32, 13751378.

Hooft, R. (1998). COLLECT. Nonius BV, Delft, The Netherlands.

Kahn-Harari, A., Ballard, R. E. \& Norris, E. K. (1973). Acta Cryst. B29, 11241126.

Marr, H. E. III, Stewart, J. M. \& Chiu, M. F. (1973). Acta Cryst. B29, 847-853.

Otwinowski, Z. \& Minor, W. (1997). Methods in Enzymology, Vol. 276, Macromolecular Crystallography, Part A, edited by C. W. Carter Jr \& R. M. Sweet, pp. 307-326. New York: Academic Press.

Sheldrick, G. M. (1997). SHELXS97 and SHELXL97. University of Göttingen, Germany.

Snaathorst, D., Doesburg, H. M., Perenboom, J. A. A. J. \& Keijzers, C. P. (1981). Inorg. Chem. 20, 2526-2532.

Sours, R. E., Fink, D. A. \& Swift, J. A. (2002). J. Am. Chem. Soc. 124, 86308636.

Spek, A. L. (1997). PLATON97. University of Utrecht, The Netherlands.

The Merck Index (2001). 13th ed. Whitehouse Station: Merck and Co. Inc.

Tuite, E. M. \& Kelly, J. M. (1993). J. Photochem. Photobiol. B, 21, 103-124. 


\section{supporting information}

Acta Cryst. (2004). E60, o1367-o1369 [https://doi.org/10.1107/S1600536804017222]

\section{3,7-Bis(dimethylamino)phenothiazin-5-ium nitrate dihydrate}

Savaridasson Jose Kavitha, Mani Mohan Raj, Krishnaswamy Panchanatheswaran and Daniel E. Lynch

3,7-Bis(dimethylamino)phenothiazin-5-ium nitrate dihydrate

Crystal data

$\mathrm{C}_{16} \mathrm{H}_{18} \mathrm{~N}_{3} \mathrm{~S}^{+} \cdot \mathrm{NO}_{3}^{-} \cdot 2 \mathrm{H}_{2} \mathrm{O}$

$M_{r}=382.44$

Triclinic, $P \overline{1}$

Hall symbol: -P 1

$a=7.6985(3) \AA$

$b=10.9638(3) \AA$

$c=11.3244$ (4) $\AA$

$\alpha=87.081(2)^{\circ}$

$\beta=76.032(2)^{\circ}$

$\gamma=73.524(2)^{\circ}$

$V=889.33(5) \AA^{3}$

\section{Data collection}

Nonius KappaCCD area-detector diffractometer

Radiation source: Nonius FR591 rotating anode Graphite monochromator

Detector resolution: 9.091 pixels $\mathrm{mm}^{-1}$

$\varphi$ and $\omega$ scans

Absorption correction: multi-scan

(SORTAV; Blessing, 1995)

$T_{\min }=0.836, T_{\max }=0.952$

Refinement

Refinement on $F^{2}$

Least-squares matrix: full

$R\left[F^{2}>2 \sigma\left(F^{2}\right)\right]=0.068$

$w R\left(F^{2}\right)=0.206$

$S=1.13$

4077 reflections

255 parameters

0 restraints

Primary atom site location: structure-invariant direct methods
$Z=2$

$F(000)=404$

$D_{\mathrm{x}}=1.428 \mathrm{Mg} \mathrm{m}^{-3}$

Mo $K \alpha$ radiation, $\lambda=0.71073 \AA$

Cell parameters from 12187 reflections

$\theta=2.9-27.5^{\circ}$

$\mu=0.22 \mathrm{~mm}^{-1}$

$T=120 \mathrm{~K}$

Prism, red

$0.42 \times 0.36 \times 0.20 \mathrm{~mm}$

17489 measured reflections

4077 independent reflections

3225 reflections with $I>2 \sigma(I)$

$R_{\text {int }}=0.068$

$\theta_{\max }=27.6^{\circ}, \theta_{\min }=3.0^{\circ}$

$h=-9 \rightarrow 9$

$k=-14 \rightarrow 14$

$l=-14 \rightarrow 14$

Secondary atom site location: difference Fourier map

Hydrogen site location: inferred from neighbouring sites

$\mathrm{H}$ atoms treated by a mixture of independent and constrained refinement

$w=1 /\left[\sigma^{2}\left(F_{\mathrm{o}}^{2}\right)+(0.0821 P)^{2}+1.7607 P\right]$ where $P=\left(F_{\mathrm{o}}{ }^{2}+2 F_{\mathrm{c}}{ }^{2}\right) / 3$

$(\Delta / \sigma)_{\max }<0.001$

$\Delta \rho_{\max }=0.64 \mathrm{e} \AA^{-3}$

$\Delta \rho_{\min }=-0.32$ e $\AA^{-3}$ 
Fractional atomic coordinates and isotropic or equivalent isotropic displacement parameters $\left(\AA^{2}\right)$

\begin{tabular}{|c|c|c|c|c|}
\hline & $x$ & $y$ & $z$ & $U_{\text {iso }} * / U_{\text {eq }}$ \\
\hline $\mathrm{C} 1$ & $0.3039(4)$ & $0.4163(3)$ & $1.2264(3)$ & $0.0253(6)$ \\
\hline H1 & 0.2291 & 0.4793 & 1.2864 & $0.032 *$ \\
\hline $\mathrm{C} 2$ & $0.4150(4)$ & $0.3079(3)$ & $1.2620(3)$ & $0.0256(6)$ \\
\hline $\mathrm{H} 2$ & 0.4170 & 0.2967 & 1.3455 & $0.032 *$ \\
\hline $\mathrm{C} 3$ & $0.5295(4)$ & $0.2101(3)$ & $1.1740(3)$ & $0.0244(6)$ \\
\hline N3 & $0.6397(4)$ & 0.1028 & $1.2068(2)$ & $0.0272(6)$ \\
\hline C31 & $0.6448(5)$ & $0.0733(3)$ & $1.3341(3)$ & $0.0345(8)$ \\
\hline H311 & 0.6506 & 0.1483 & 1.3749 & $0.043 *$ \\
\hline H312 & 0.7550 & 0.0025 & 1.3368 & $0.043 *$ \\
\hline H313 & 0.5321 & 0.0495 & 1.3756 & $0.043 *$ \\
\hline $\mathrm{C} 32$ & $0.7498(5)$ & 0.0020 & $1.1173(3)$ & $0.0356(8)$ \\
\hline H321 & 0.6679 & -0.0213 & 1.0738 & $0.045^{*}$ \\
\hline H322 & 0.8106 & -0.0726 & 1.1590 & $0.045 *$ \\
\hline H323 & 0.8448 & 0.0322 & 1.0592 & $0.045^{*}$ \\
\hline $\mathrm{C} 4$ & $0.5233(4)$ & 0.2303 & $1.0504(3)$ & $0.0245(6)$ \\
\hline $\mathrm{H} 4$ & 0.5982 & 0.1670 & 0.9908 & $0.031 *$ \\
\hline S5 & $0.40954(11)$ & $0.35433(7)$ & $0.86311(7)$ & $0.0265(2)$ \\
\hline C6 & $0.2292(4)$ & 0.5433 & $0.7434(3)$ & $0.0241(6)$ \\
\hline H6 & 0.2934 & 0.4895 & 0.6741 & $0.030 *$ \\
\hline $\mathrm{C} 7$ & $0.1059(4)$ & $0.6632(3)$ & $0.7289(3)$ & $0.0239(6)$ \\
\hline N7 & $0.0768(4)$ & $0.6987(3)$ & $0.6188(2)$ & $0.0278(6)$ \\
\hline C71 & $0.1732(6)$ & $0.6157(3)$ & $0.5125(3)$ & $0.0372(8)$ \\
\hline H711 & 0.3070 & 0.6078 & 0.4951 & $0.046^{*}$ \\
\hline H712 & 0.1244 & 0.6520 & 0.4422 & $0.046 *$ \\
\hline H713 & 0.1531 & 0.5315 & 0.5287 & $0.046^{*}$ \\
\hline $\mathrm{C} 72$ & $-0.0522(5)$ & 0.8200 & $0.5977(3)$ & $0.0343(8)$ \\
\hline H721 & -0.1634 & 0.8390 & 0.6653 & $0.043 *$ \\
\hline H722 & -0.0887 & 0.8143 & 0.5215 & $0.043 *$ \\
\hline H723 & 0.0091 & 0.8879 & 0.5923 & $0.043 *$ \\
\hline $\mathrm{C} 8$ & $0.0147(4)$ & $0.7430(3)$ & $0.8354(3)$ & $0.0258(7)$ \\
\hline H8 & -0.0662 & 0.8250 & 0.8282 & $0.032 *$ \\
\hline C9 & $0.0430(5)$ & $0.7022(3)$ & $0.9465(3)$ & $0.0263(7)$ \\
\hline H9 & -0.0202 & 0.7566 & 1.0155 & $0.033 *$ \\
\hline N10 & $0.1816(4)$ & $0.5488(2)$ & $1.0768(2)$ & $0.0232(5)$ \\
\hline $\mathrm{C} 11$ & $0.2953(4)$ & $0.4392(3)$ & $1.1023(3)$ & $0.0226(6)$ \\
\hline $\mathrm{C} 12$ & $0.4104(4)$ & $0.3402(3)$ & $1.0157(3)$ & $0.0231(6)$ \\
\hline $\mathrm{C} 13$ & $0.2576(4)$ & $0.5031(3)$ & $0.8561(3)$ & $0.0216(6)$ \\
\hline $\mathrm{C} 14$ & $0.1637(4)$ & 0.5808 & $0.9632(3)$ & $0.0223(6)$ \\
\hline N1 & $0.7431(4)$ & $0.0916(3)$ & $0.7386(3)$ & $0.0341(7)$ \\
\hline $\mathrm{O} 1$ & $0.7351(4)$ & 0.1430 & $0.6394(3)$ & $0.0543(8)$ \\
\hline $\mathrm{O} 2$ & $0.8749(4)$ & 0.0886 & $0.7856(3)$ & $0.0531(8)$ \\
\hline $\mathrm{O} 3$ & $0.6206(5)$ & 0.0440 & $0.7940(3)$ & $0.0568(9)$ \\
\hline $\mathrm{O} 1 \mathrm{~W}$ & $0.0499(4)$ & 0.2807 & $0.7190(2)$ & $0.0354(6)$ \\
\hline H11W & $-0.012(6)$ & $0.223(5)$ & $0.738(4)$ & $0.043(12) *$ \\
\hline H12W & $-0.022(7)$ & $0.334(5)$ & $0.772(5)$ & $0.049(13)^{*}$ \\
\hline
\end{tabular}




\begin{tabular}{lllll}
$\mathrm{O} 2 \mathrm{~W}$ & $0.3966(5)$ & $0.2779(3)$ & $0.5684(2)$ & $0.0390(6)$ \\
$\mathrm{H} 21 \mathrm{~W}$ & $0.471(8)$ & $0.225(5)$ & $0.597(5)$ & $0.058(16)^{*}$ \\
$\mathrm{H} 22 \mathrm{~W}$ & $0.306(7)$ & $0.271(5)$ & $0.606(5)$ & $0.044(14)^{*}$ \\
\hline
\end{tabular}

Atomic displacement parameters $\left(\AA^{2}\right)$

\begin{tabular}{lllllll}
\hline & $U^{11}$ & $U^{22}$ & $U^{33}$ & $U^{12}$ & $U^{13}$ & $U^{23}$ \\
\hline C1 & $0.0292(16)$ & $0.0240(15)$ & $0.0211(14)$ & $-0.0060(13)$ & $-0.0038(12)$ & $-0.0036(11)$ \\
C2 & $0.0275(16)$ & $0.0290(16)$ & $0.0206(14)$ & $-0.0081(13)$ & $-0.0058(12)$ & $0.0002(12)$ \\
C3 & $0.0210(15)$ & $0.0255(15)$ & $0.0258(15)$ & $-0.0064(12)$ & $-0.0042(12)$ & $0.0019(12)$ \\
N3 & $0.0273(14)$ & $0.0271(14)$ & $0.0242(13)$ & $-0.0021(11)$ & $-0.0071(11)$ & $0.0013(10)$ \\
C31 & $0.0397(19)$ & $0.0335(18)$ & $0.0248(16)$ & $-0.0006(15)$ & $-0.0098(14)$ & $0.0040(13)$ \\
C32 & $0.0344(19)$ & $0.0325(18)$ & $0.0309(17)$ & $0.0059(14)$ & $-0.0084(14)$ & $-0.0015(14)$ \\
C4 & $0.0225(15)$ & $0.0238(15)$ & $0.0235(15)$ & $-0.0022(12)$ & $-0.0028(12)$ & $-0.0022(11)$ \\
S5 & $0.0305(4)$ & $0.0231(4)$ & $0.0199(4)$ & $0.0013(3)$ & $-0.0048(3)$ & $-0.0022(3)$ \\
C6 & $0.0260(15)$ & $0.0231(15)$ & $0.0222(14)$ & $-0.0063(12)$ & $-0.0041(12)$ & $-0.0016(11)$ \\
C7 & $0.0243(15)$ & $0.0243(15)$ & $0.0246(15)$ & $-0.0089(12)$ & $-0.0064(12)$ & $0.0022(12)$ \\
N7 & $0.0320(15)$ & $0.0231(13)$ & $0.0250(13)$ & $-0.0012(11)$ & $-0.0080(11)$ & $0.0003(10)$ \\
C71 & $0.054(2)$ & $0.0296(17)$ & $0.0205(15)$ & $0.0014(16)$ & $-0.0101(15)$ & $-0.0008(13)$ \\
C72 & $0.0382(19)$ & $0.0294(17)$ & $0.0303(17)$ & $-0.0006(14)$ & $-0.0100(14)$ & $0.0032(13)$ \\
C8 & $0.0260(16)$ & $0.0210(15)$ & $0.0286(16)$ & $-0.0036(12)$ & $-0.0065(12)$ & $0.0001(12)$ \\
C9 & $0.0283(16)$ & $0.0228(15)$ & $0.0255(15)$ & $-0.0047(12)$ & $-0.0040(12)$ & $-0.0049(12)$ \\
N10 & $0.0253(13)$ & $0.0215(12)$ & $0.0224(12)$ & $-0.0059(10)$ & $-0.0052(10)$ & $-0.0023(10)$ \\
C11 & $0.0229(15)$ & $0.0217(14)$ & $0.0234(14)$ & $-0.0072(12)$ & $-0.0040(11)$ & $-0.0015(11)$ \\
C12 & $0.0233(15)$ & $0.0249(15)$ & $0.0212(14)$ & $-0.0088(12)$ & $-0.0028(11)$ & $-0.0013(11)$ \\
C13 & $0.0221(14)$ & $0.0200(14)$ & $0.0223(14)$ & $-0.0054(11)$ & $-0.0049(11)$ & $-0.0010(11)$ \\
C14 & $0.0231(15)$ & $0.0211(14)$ & $0.0228(14)$ & $-0.0062(12)$ & $-0.0053(11)$ & $-0.0006(11)$ \\
N1 & $0.0309(15)$ & $0.0285(15)$ & $0.0371(16)$ & $-0.0040(12)$ & $-0.0014(13)$ & $-0.0032(12)$ \\
O1 & $0.0538(18)$ & $0.0563(19)$ & $0.0371(15)$ & $0.0022(15)$ & $-0.0042(13)$ & $0.0103(13)$ \\
O2 & $0.0501(18)$ & $0.0491(18)$ & $0.064(2)$ & $-0.0109(14)$ & $-0.0253(15)$ & $0.0024(15)$ \\
O3 & $0.0553(19)$ & $0.0553(19)$ & $0.0579(19)$ & $-0.0301(16)$ & $0.0095(15)$ & $-0.0096(15)$ \\
O1W & $0.0364(14)$ & $0.0331(14)$ & $0.0320(13)$ & $-0.0022(12)$ & $-0.0066(11)$ & $-0.0059(11)$ \\
O2W & $0.0415(17)$ & $0.0415(16)$ & $0.0281(13)$ & $-0.0048(13)$ & $-0.0054(13)$ & $0.0017(11)$ \\
& & & & & & \\
& & & & & &
\end{tabular}

Geometric parameters $\left(\AA,{ }^{\circ}\right)$

\begin{tabular}{llll}
\hline $\mathrm{C} 1-\mathrm{C} 2$ & $1.359(4)$ & $\mathrm{N} 7-\mathrm{C} 71$ & $1.458(4)$ \\
$\mathrm{C} 1-\mathrm{C} 11$ & $1.430(4)$ & $\mathrm{N} 7-\mathrm{C} 72$ & $1.464(4)$ \\
$\mathrm{C} 1-\mathrm{H} 1$ & 0.95 & $\mathrm{C} 71-\mathrm{H} 711$ & 0.98 \\
$\mathrm{C} 2-\mathrm{C} 3$ & $1.439(4)$ & $\mathrm{C} 71-\mathrm{H} 712$ & 0.98 \\
$\mathrm{C} 2-\mathrm{H} 2$ & 0.95 & $\mathrm{C} 71-\mathrm{H} 713$ & 0.98 \\
$\mathrm{C} 3-\mathrm{N} 3$ & $1.335(4)$ & $\mathrm{C} 72-\mathrm{H} 721$ & 0.98 \\
$\mathrm{C} 3-\mathrm{C} 4$ & $1.416(4)$ & $\mathrm{C} 72-\mathrm{H} 722$ & 0.98 \\
$\mathrm{~N} 3-\mathrm{C} 32$ & $1.461(4)$ & $\mathrm{C} 72-\mathrm{H} 723$ & 0.98 \\
$\mathrm{~N} 3-\mathrm{C} 31$ & $1.469(4)$ & $\mathrm{C} 8-\mathrm{C} 9$ & $1.361(4)$ \\
$\mathrm{C} 31-\mathrm{H} 311$ & 0.98 & $\mathrm{C} 8-\mathrm{H} 8$ & 0.95 \\
$\mathrm{C} 31-\mathrm{H} 312$ & 0.98 & $\mathrm{C} 9-\mathrm{C} 14$ & $1.424(4)$ \\
$\mathrm{C} 31-\mathrm{H} 313$ & 0.98 & $\mathrm{C} 9-\mathrm{H} 9$ & 0.95
\end{tabular}




\begin{tabular}{|c|c|c|c|}
\hline $\mathrm{C} 32-\mathrm{H} 321$ & 0.98 & $\mathrm{~N} 10-\mathrm{C} 11$ & $1.335(4)$ \\
\hline C $32-\mathrm{H} 322$ & 0.98 & $\mathrm{~N} 10-\mathrm{C} 14$ & $1.344(4)$ \\
\hline $\mathrm{C} 32-\mathrm{H} 323$ & 0.98 & $\mathrm{C} 11-\mathrm{C} 12$ & $1.438(4)$ \\
\hline $\mathrm{C} 4-\mathrm{C} 12$ & $1.373(4)$ & $\mathrm{C} 13-\mathrm{C} 14$ & $1.432(4)$ \\
\hline $\mathrm{C} 4-\mathrm{H} 4$ & 0.95 & $\mathrm{~N} 1-\mathrm{O} 3$ & $1.231(4)$ \\
\hline $\mathrm{S} 5-\mathrm{C} 13$ & $1.728(3)$ & $\mathrm{N} 1-\mathrm{O} 1$ & $1.238(4)$ \\
\hline $\mathrm{S} 5-\mathrm{C} 12$ & $1.728(3)$ & $\mathrm{N} 1-\mathrm{O} 2$ & $1.246(4)$ \\
\hline $\mathrm{C} 6-\mathrm{C} 13$ & $1.376(4)$ & $\mathrm{O} 1 \mathrm{~W}-\mathrm{H} 11 \mathrm{~W}$ & $0.88(5)$ \\
\hline $\mathrm{C} 6-\mathrm{C} 7$ & $1.415(4)$ & $\mathrm{O} 1 \mathrm{~W}-\mathrm{H} 12 \mathrm{~W}$ & $0.84(5)$ \\
\hline $\mathrm{C} 6-\mathrm{H} 6$ & 0.95 & $\mathrm{O} 2 \mathrm{~W}-\mathrm{H} 21 \mathrm{~W}$ & $0.81(6)$ \\
\hline $\mathrm{C} 7-\mathrm{N} 7$ & $1.340(4)$ & $\mathrm{O} 2 \mathrm{~W}-\mathrm{H} 22 \mathrm{~W}$ & $0.75(5)$ \\
\hline $\mathrm{C} 7-\mathrm{C} 8$ & $1.436(4)$ & & \\
\hline $\mathrm{C} 2-\mathrm{C} 1-\mathrm{C} 11$ & $122.5(3)$ & C71-H711-109.5 & \\
\hline $\mathrm{C} 2-\mathrm{C} 1-\mathrm{H} 1$ & 118.7 & $\mathrm{C} 71-\mathrm{H} 712-109.5$ & \\
\hline $\mathrm{C} 11-\mathrm{C} 1-\mathrm{H} 1$ & 118.7 & C71-H712-109.5 & \\
\hline $\mathrm{C} 1-\mathrm{C} 2-\mathrm{C} 3$ & $120.3(3)$ & C71-H713-109.5 & \\
\hline $\mathrm{C} 1-\mathrm{C} 2-\mathrm{H} 2$ & 119.8 & C71-H713-109.5 & \\
\hline $\mathrm{C} 3-\mathrm{C} 2-\mathrm{H} 2$ & 119.8 & C71-H713-109.5 & \\
\hline $\mathrm{N} 3-\mathrm{C} 3-\mathrm{C} 4$ & $120.3(3)$ & C72-H721-109.5 & \\
\hline $\mathrm{N} 3-\mathrm{C} 3-\mathrm{C} 2$ & $121.4(3)$ & $\mathrm{C} 72-\mathrm{H} 722-109.5$ & \\
\hline $\mathrm{C} 4-\mathrm{C} 3-\mathrm{C} 2$ & $118.3(3)$ & $\mathrm{C} 72-\mathrm{H} 722-109.5$ & \\
\hline $\mathrm{C} 3-\mathrm{N} 3-\mathrm{C} 32$ & $120.9(3)$ & $\mathrm{C} 72-\mathrm{H} 723-109.5$ & \\
\hline $\mathrm{C} 3-\mathrm{N} 3-\mathrm{C} 31$ & $122.9(3)$ & $\mathrm{C} 72-\mathrm{H} 723-109.5$ & \\
\hline $\mathrm{C} 32-\mathrm{N} 3-\mathrm{C} 31$ & $115.9(3)$ & $\mathrm{C} 72-\mathrm{H} 723-109.5$ & \\
\hline $\mathrm{N} 3-\mathrm{C} 31-\mathrm{H} 311$ & 109.5 & $\mathrm{C} 8-\mathrm{C} 7-120.6(3)$ & \\
\hline $\mathrm{N} 3-\mathrm{C} 31-\mathrm{H} 312$ & 109.5 & $\mathrm{C} 8-\mathrm{H} 8-119.7$ & \\
\hline $\mathrm{H} 311-\mathrm{C} 31-\mathrm{H} 312$ & 109.5 & $\mathrm{C} 8-\mathrm{H} 8-119.7$ & \\
\hline $\mathrm{N} 3-\mathrm{C} 31-\mathrm{H} 313$ & 109.5 & $\mathrm{C} 9-\mathrm{C} 14-122.4(3)$ & \\
\hline $\mathrm{H} 311-\mathrm{C} 31-\mathrm{H} 313$ & 109.5 & C9-H9-118.8 & \\
\hline $\mathrm{H} 312-\mathrm{C} 31-\mathrm{H} 313$ & 109.5 & C9-H9-118.8 & \\
\hline $\mathrm{N} 3-\mathrm{C} 32-\mathrm{H} 321$ & 109.5 & $\mathrm{~N} 10-\mathrm{C} 14-123.0(3)$ & \\
\hline N3-C32-H322 & 109.5 & $\mathrm{C} 11-\mathrm{C} 1-117.9(3)$ & \\
\hline $\mathrm{H} 321-\mathrm{C} 32-\mathrm{H} 322$ & 109.5 & $\mathrm{C} 11-\mathrm{C} 12-125.8(3)$ & \\
\hline $\mathrm{N} 3-\mathrm{C} 32-\mathrm{H} 323$ & 109.5 & $\mathrm{C} 11-\mathrm{C} 12-116.3(3)$ & \\
\hline $\mathrm{H} 321-\mathrm{C} 32-\mathrm{H} 323$ & 109.5 & C12-C11-121.7 (3) & \\
\hline $\mathrm{H} 322-\mathrm{C} 32-\mathrm{H} 323$ & 109.5 & $\mathrm{C} 12-\mathrm{S} 5-117.2(2)$ & \\
\hline $\mathrm{C} 12-\mathrm{C} 4-\mathrm{C} 3$ & $120.8(3)$ & $\mathrm{C} 12-\mathrm{S} 5-121.0(2)$ & \\
\hline $\mathrm{C} 12-\mathrm{C} 4-\mathrm{H} 4$ & 119.6 & $\mathrm{C} 13-\mathrm{C} 14-121.4(3)$ & \\
\hline $\mathrm{C} 3-\mathrm{C} 4-\mathrm{H} 4$ & 119.6 & $\mathrm{C} 13-\mathrm{S} 5-117.1$ (2) & \\
\hline $\mathrm{C} 13-\mathrm{S} 5-\mathrm{C} 12$ & $103.28(15)$ & $\mathrm{C} 13-\mathrm{S} 5-121.4(2)$ & \\
\hline $\mathrm{C} 13-\mathrm{C} 6-\mathrm{C} 7$ & $121.2(3)$ & $\mathrm{C} 14-\mathrm{C} 9-118.0(3)$ & \\
\hline $\mathrm{C} 13-\mathrm{C} 6-\mathrm{H} 6$ & 119.4 & $\mathrm{C} 14-\mathrm{C} 13-125.4(3)$ & \\
\hline $\mathrm{C} 7-\mathrm{C} 6-\mathrm{H} 6$ & 119.4 & $\mathrm{C} 14-\mathrm{C} 13-116.6(3)$ & \\
\hline $\mathrm{N} 7-\mathrm{C} 7-\mathrm{C} 6$ & $120.0(3)$ & $\mathrm{N} 1-\mathrm{O} 1-121.1(4)$ & \\
\hline $\mathrm{N} 7-\mathrm{C} 7-\mathrm{C} 8$ & $122.2(3)$ & $\mathrm{N} 1-\mathrm{O} 2-118.4(3)$ & \\
\hline $\mathrm{C} 6-\mathrm{C} 7-\mathrm{C} 8$ & $117.8(3)$ & $\mathrm{N} 1-\mathrm{O} 2-120.5(3)$ & \\
\hline $\mathrm{C} 7-\mathrm{N} 7-\mathrm{C} 71$ & $120.7(3)$ & $\mathrm{O} 1 \mathrm{~W}-\mathrm{H} 12 \mathrm{~W}-95(4)$ & \\
\hline
\end{tabular}




$\begin{array}{ll}\mathrm{C} 7-\mathrm{N} 7-\mathrm{C} 72 & 122.9(3) \\ \mathrm{N} 7-\mathrm{C} 72-116.4(3) & \\ \mathrm{C} 11-\mathrm{C} 1-\mathrm{C} 2-\mathrm{C} 3 & -0.3(5) \\ \mathrm{C} 1-\mathrm{C} 2-\mathrm{C} 3-\mathrm{N} 3 & -180.0(3) \\ \mathrm{C} 1-\mathrm{C} 2-\mathrm{C} 3-\mathrm{C} 4 & 0.2(5) \\ \mathrm{C} 4-\mathrm{C} 3-\mathrm{N} 3-\mathrm{C} 32 & -2.6(5) \\ \mathrm{C} 2-\mathrm{C} 3-\mathrm{N} 3-\mathrm{C} 32 & 177.6(3) \\ \mathrm{C} 4-\mathrm{C} 3-\mathrm{N} 3-\mathrm{C} 31 & -176.6(3) \\ \mathrm{C} 2-\mathrm{C} 3-\mathrm{N} 3-\mathrm{C} 31 & 3.5(5) \\ \mathrm{N} 3-\mathrm{C} 3-\mathrm{C} 4-\mathrm{C} 12 & 179.9(3) \\ \mathrm{C} 2-\mathrm{C} 3-\mathrm{C} 4-\mathrm{C} 12 & -0.2(5) \\ \mathrm{C} 13-\mathrm{C} 6-\mathrm{C} 7-\mathrm{N} 7 & -178.2(3) \\ \mathrm{C} 13-\mathrm{C} 6-\mathrm{C} 7-\mathrm{C} 8 & 1.4(5) \\ \mathrm{C} 6-\mathrm{C} 7-\mathrm{N} 7-\mathrm{C} 71 & -1.0(5) \\ \mathrm{C} 8-\mathrm{C} 7-\mathrm{N} 7-\mathrm{C} 71 & 179.4(3) \\ \mathrm{C} 6-\mathrm{C} 7-\mathrm{N} 7-\mathrm{C} 72 & 178.5(3) \\ \mathrm{C} 8-\mathrm{C} 7-\mathrm{N} 7-\mathrm{C} 72 & -1.2(5) \\ \mathrm{N} 7-\mathrm{C} 7-\mathrm{C} 8-\mathrm{C} 9 & 177.9(3) \\ \mathrm{C} 6-\mathrm{C} 7-\mathrm{C} 8-\mathrm{C} 9 & -1.8(5) \\ \mathrm{C} 7-\mathrm{C} 8-\mathrm{C} 9-\mathrm{C} 14 & 0.6(5) \\ \mathrm{C} 14-\mathrm{N} 10-\mathrm{C} 11-\mathrm{C} 1 & 179.3(3) \\ \mathrm{C} 14-\mathrm{N} 10-\mathrm{C} 11-\mathrm{C} 12 & -1.2(5) \\ \mathrm{C} 2-\mathrm{C} 1-\mathrm{C} 11-\mathrm{N} 10 & 180.0(3)\end{array}$

$\mathrm{O} 2 \mathrm{~W}-\mathrm{H} 22 \mathrm{~W}-102(5)$

$\begin{array}{ll}\mathrm{C} 2-\mathrm{C} 1-\mathrm{C} 11-\mathrm{C} 12 & 0.4(5) \\ \mathrm{C} 3-\mathrm{C} 4-\mathrm{C} 12-\mathrm{C} 11 & 0.4(5) \\ \mathrm{C} 3-\mathrm{C} 4-\mathrm{C} 12-\mathrm{S} 5 & -178.8(2) \\ \mathrm{N} 10-\mathrm{C} 11-\mathrm{C} 12-\mathrm{C} 4 & -180.0(3) \\ \mathrm{C} 1-\mathrm{C} 11-\mathrm{C} 12-\mathrm{C} 4 & -0.5(4) \\ \mathrm{N} 10-\mathrm{C} 11-\mathrm{C} 12-\mathrm{S} 5 & -0.8(4) \\ \mathrm{C} 1-\mathrm{C} 11-\mathrm{C} 12-\mathrm{S} 5 & 178.7(2) \\ \mathrm{C} 13-\mathrm{S} 5-\mathrm{C} 12-\mathrm{C} 4 & -178.9(2) \\ \mathrm{C} 13-\mathrm{S} 5-\mathrm{C} 12-\mathrm{C} 11 & 1.9(3) \\ \mathrm{C} 7-\mathrm{C} 6-\mathrm{C} 13-\mathrm{C} 14 & 0.1(5) \\ \mathrm{C} 7-\mathrm{C} 6-\mathrm{C} 13-\mathrm{S} 5 & 179.4(2) \\ \mathrm{C} 12-\mathrm{S} 5-\mathrm{C} 13-\mathrm{C} 6 & 179.2(2) \\ \mathrm{C} 12-\mathrm{S} 5-\mathrm{C} 13-\mathrm{C} 14 & -1.5(3) \\ \mathrm{C} 11-\mathrm{N} 10-\mathrm{C} 14-\mathrm{C} 9 & -177.9(3) \\ \mathrm{C} 11-\mathrm{N} 10-\mathrm{C} 14-\mathrm{C} 13 & 1.6(5) \\ \mathrm{C} 8-\mathrm{C} 9-\mathrm{C} 14-\mathrm{N} 10 & -179.6(3) \\ \mathrm{C} 8-\mathrm{C} 9-\mathrm{C} 14-\mathrm{C} 13 & 0.9(5) \\ \mathrm{C} 6-\mathrm{C} 13-\mathrm{C} 14-\mathrm{N} 10 & 179.3(3) \\ \mathrm{S} 5-\mathrm{C} 13-\mathrm{C} 14-\mathrm{N} 10 & 0.0(4) \\ \mathrm{C} 6-\mathrm{C} 13-\mathrm{C} 14-\mathrm{C} 9 & -1.2(4) \\ \mathrm{S} 5-\mathrm{C} 13-\mathrm{C} 14-\mathrm{C} 9 & 179.5(2)\end{array}$

Hydrogen-bond geometry $\left(\AA,{ }^{\circ}\right)$

\begin{tabular}{lllll}
\hline$D-\mathrm{H} \cdots A$ & $D-\mathrm{H}$ & $\mathrm{H} \cdots A$ & $D \cdots A$ & $D-\mathrm{H} \cdots A$ \\
\hline $\mathrm{O} 1 W-\mathrm{H} 11 W^{\cdots} \cdot \mathrm{O} 2^{\mathrm{i}}$ & $0.88(5)$ & $1.91(5)$ & $2.789(4)$ & $174(4)$ \\
$\mathrm{O} 1 W-\mathrm{H} 12 W \cdots \mathrm{N} 10^{\mathrm{ii}}$ & $0.84(5)$ & $2.10(5)$ & $2.929(4)$ & $172(4)$ \\
$\mathrm{O} 2 W-\mathrm{H} 21 W \cdots \mathrm{O} 1$ & $0.81(6)$ & $2.14(6)$ & $2.886(5)$ & $154(5)$ \\
$\mathrm{O} 2 W-\mathrm{H} 22 W \cdots \mathrm{O} 1 W$ & $0.75(5)$ & $2.05(5)$ & $2.792(4)$ & $171(5)$ \\
\hline
\end{tabular}

Symmetry codes: (i) $x-1, y, z$; (ii) $-x,-y+1,-z+2$. 